\title{
Colour strings, Pomerons and Color Glass Condensate
}

\section{Elena Ferreiro*}

Departamento de Fisica de Particulas, Universidade de Santiago de Compostela, Spain

E-mail: elena@fpaxp1.usc.es

In the recent experiments like DIS at HERA or the heavy-ion experiments at RHIC, and also in expected LHC at CERN, the number of involved partons is very large, due to the high energy and/or the high number of participants of those experiments. These high parton densities should in principal lead to an extremely huge multiparticle production, but experimentally we have seen that this is not the case. So there should be a mechanism that reduces the number of created particle. Here, I review the problem of parton saturation and its implications through three in principal different approaches, but somewhat related: saturation in a geometrical approach, QCD saturation through the Color Glass Condensate and perturbative Pomeron approach with initial conditions.

International Europhysics Conference on High Energy Physics

July 21st - 27th 2005

Lisboa, Portugal

* Speaker. 


\section{Geometrical approach to saturation: String models and percolation}

In many models of hadronic and nuclear collisions, color strings are exchanged between the projectile and the target. Those strings act as color sources of particles through the creation of $q-\bar{q}$ pairs from the sea. The number of strings grows with the energy and with the number of nucleons of the participant nuclei.

In impact parameter space these strings are seen as circles inside the total collision area. When the density of strings becomes high the string color fields begin to overlap and eventually individual strings may fuse, forming a new string -cluster- which has a higher color charge at its ends, corresponding to the summation of the color charges located at the ends of the original strings. The new string clusters break into hadrons according to their higher color. As a result, there is a reduction of the total multiplicity. Also, as the energy-momenta of the original strings are summed to obtain the energy-momentum of the resulting cluster, the mean transverse momentum of the particles created by those clusters is increased compared to the one of the particles created from individual sources.

As the number of strings increases, more strings overlap. Some years ago, it has been proposed in Ref. [1] that above a critical density of strings percolation occurs, so that paths of overlapping circles are formed through the whole collision area. Along these paths the medium behaves like a color conductor. Also in [1], we have made the remark that several fused strings can be considered as a domain of a non thermalized Quark Gluon Plasma. The percolation gives rise to the formation of a non thermalized Quark Gluon Plasma on a nuclear scale.

Note that here we are not speaking about a final state interaction phenomenon, since there is no thermalization involved. In fact, what we are trying is to determine under what conditions the initial state configurations can lead to color connection, and more specifically, if variations of the initial state can lead to a transition from disconnected to connected color clusters. The formalism underlying the transition from disconnected to connected systems is given by percolation theory, which describes geometric critical behavior.

Consider placing $N$ small circular discs (color sources, strings or partons) of radius $r$ onto a large circular manifold (the transverse nuclear plane) of radius $R$; the small discs may overlap. With increasing density

$$
\eta=\frac{N \pi r^{2}}{\pi R^{2}}
$$

this overlap will lead to more and larger connected clusters. The most interesting feature of this phenomenon is that the average cluster size increases very suddenly from very small to very large values. This suggests some kind of geometric critical behavior. In fact, the cluster size diverges at a critical threshold value $\eta_{c}$ of the density. This appearance of an infinite cluster at $\eta=\eta_{c}$ is defined as percolation: the size of the cluster reaches the size of the system. $\eta_{c}$ has been computed using Monte Carlo simulation, direct connectedness expansion and other different methods. All the results are in the range $\eta_{c}=1.12 \div 1.175$.

In our model [1] we had proposed a fixed radius for the independent color sources of $r=$ $0.2 \div 0.25 \mathrm{fm}$, that corresponds to a momentum around $1 \mathrm{GeV}$. According to eq. (1.1), in order to estimate the density $\eta$, one needs to know the number of sources $N$. It is obtained from the String Fusion Model Code (SFMC) [2]. Note that $N$ will depend on the energy $\sqrt{s}$ (or equivalently, on $x$ ) and on the number of participant nucleons $A$, so in some way the condition to achieve percolation 
depends on $A$ and $s, \eta=\eta(A, x) . \pi R^{2}$ corresponds simply to the nuclear overlap area, $S_{A}$, at the given impact parameter. That leads to the following results: at SPS energies, the critical threshold for percolation could eventually been achieved for the most central $\mathrm{Pb}-\mathrm{Pb}$ collisions, and for sure in Au-Au central collisions at RHIC energies and even in p-p collisions at LHC energies. Just as an example, in first approximation, one can estimates analytically that at very high energies, for central A-A collisions, if we take the number of initially created sources as proportional to the number of collisions, $N \propto A^{4 / 3}$ and the nuclear overlap area $S_{A}=\pi R^{2} \propto A^{2 / 3}$ then

$$
\eta=\frac{N \pi r^{2}}{\pi R^{2}}=\frac{N_{\text {sources }} S_{1}}{S_{A}} \propto A^{2 / 3} .
$$

\section{The Color Glass Condensate}

Now we arrive to another approach, the QCD saturation through the formation of a Color Glass Condensate (CGC) [3]. The idea is the following: At high energy, the QCD cross-sections are controlled by small longitudinal momentum gluons in the hadron wave function, whose density grows rapidly with increasing energy or decreasing $x$, due to the enhancement of radiative process. If one applies perturbation theory to this regime, one finds that, by resumming dominant radiative corrections at high energy, the BFKL equation -linear- leads to a gluon density that grows like a power of $s$ and in consequence to a cross-section that violates the Froissart bound. In fact, with increasing energy, recombination effects -that are non-linear-favored by the high density of gluons should become more important and lead to an eventual saturation of parton densities.

These effects become important when the interaction probability for the gluons becomes of order one. Taking $\frac{\alpha_{s} N_{c}}{Q^{2}}$ as the transverse size of the gluon and $\frac{x G\left(x, Q^{2}\right)}{\pi R^{2}}$ as the density of gluons, the interaction probability is expressed by

$$
\frac{\alpha_{s} N_{c}}{Q^{2}} \times \frac{x G\left(x, Q^{2}\right)}{\pi R^{2}} .
$$

Equivalently, for a given energy, saturation occurs for those gluons having a sufficiently large transverse size $r_{\perp}^{2} \sim 1 / Q^{2}$, larger than some critical value $1 / Q_{s}(x, A)$. So the phenomenon of saturation introduces a characteristic momentum scale, the saturation momentum $Q_{s}(x, A)$, which is a measure of the density of the saturated gluons, and grows rapidly with $1 / x$ and $A$ (the atomic number). The probability of interaction -that can be understood as "overlapping" of the gluons in the transverse space- becomes of order one for those gluons with momenta $Q^{2} \lesssim Q_{s}(x, A)$ where

$$
Q_{s}^{2}(x, A)=\alpha_{s} N_{c} \frac{x G\left(x, Q_{s}^{2}\right)}{\pi R^{2}} \equiv \frac{(\text { color charge })^{2}}{\text { area }} .
$$

For a nucleus, $x G_{A}\left(x, Q_{s}^{2}\right) \propto A$ and $\pi R_{A}^{2} \propto A^{2 / 3}$, so eq. (2.2) predicts $Q_{s}^{2} \propto A^{1 / 3}$. One can estimate the saturation scale by inserting the BFKL approximation into eq. (2.2). This gives (with $\delta \approx 1 / 3$ and $\lambda \approx c \bar{\alpha}_{s}$ in a first approximation):

$$
Q_{s}^{2}(x, A) \sim A^{\delta} x^{-\lambda},
$$

which indicates that an efficient way to create a high-density environment is to combine large nuclei with moderately small values of $x$, as it is done at RHIC. In fact the estimated momentum for saturation at RHIC will be $Q_{s}=1 \div 2 \mathrm{GeV}$, in accordance with the result of the previous section. 


\section{Perturbative QCD pomeron with saturation in the initial conditions}

Let us now try a different approach [4]. Consider now the nucleus-nucleus interaction as governed by the exchange of pomerons. Its propagation is governed by the BFKL equation. Its interaction is realized by the triple pomeron vertex. Knowing that the AGK rules are satisfied for the diagrams with BFKL pomerons interacting via the triple pomeron vertex one can conclude that the inclusive cross-section will be given by the convolution of two sums of fan diagrams propagating from the emitted particle towards the two nuclei. Taking $A=B$ and constant nuclear density for $|b|<R_{A}$, one can find the inclusive cross-section in perturbative QCD as

$$
I_{A}(y, k)=A^{2 / 3} \pi R_{0}^{2} \frac{8 N_{c} \alpha_{s}}{k^{2}} \int d^{2} r e^{i k r}\left[\Delta \Phi_{A}(Y-y, r)\right]\left[\Delta \Phi_{A}(y, r)\right]
$$

where $\Delta$ is the two-dimensional Laplacian and $\Phi(y, r)$ is the sum of all fan diagrams connecting the pomeron at rapidity $y$ and of the transverse dimension $r$ with the colliding nuclei, one at rest and the other at rapidity $Y$. The function $\phi_{A}(y, r)=\Phi(y, r) /\left(2 \pi r^{2}\right)$, in the momentum space, satisfies the well-known non-linear Balitsky-Kovchegov equation

$$
\frac{\partial \phi(y, q)}{\partial \bar{y}}=-H \phi(y, q)-\phi^{2}(y, q)
$$

where $\bar{y}=\bar{\alpha} y, \bar{\alpha}=\alpha_{s} N_{c} / \pi, \alpha_{s}$ and $N_{c}$ are the strong coupling constant and the number of colors, respectively, and $H$ is the BFKL Hamiltonian. This equation has to be solved with the initial condition at $y=0$ determined by the color dipole distribution in the nucleon smeared by the profile function of the nucleus.

We can take the initial condition in accordance with the Golec-Biernat distribution [5], that takes into account saturation according to the CGC. Then, one can observe that whereas at relatively small momenta the inclusive cross-sections are proportional to $A$, that is to the number of participants, at larger momenta they grow with $A$ faster, however noticeably slowlier than the number of collisions, approximately as $A^{1.1}$. The interval of momenta for which $I_{A} \propto A$ is growing with energy.

We have compared different models that takes into account saturation in different ways: from the semi-phenomenological fusing color sources picture for the soft domain including percolation, the QCD saturation through the Color Glass Condensate and those which follow from the pomeron approach, perturbatively derived from QCD, taking into account saturation in the initial conditions. In fact, it seems that the exchanged of elemental objects, color sources -strings, partons or pomerons-, should lead to a saturation in the initial conditions when the densities are high enough.

\section{References}

[1] N. Armesto, M. A. Braun, E. G. Ferreiro and C. Pajares, Phys. Rev. Lett. 77, 3736 (1996).

[2] N. S. Amelin, M. A. Braun and C. Pajares, Phys. Lett. B 306, 312 (1993).

[3] E. Iancu, A. Leonidov and L. McLerran, Nucl. Phys. A 692 (2001), 583; E. Ferreiro, E. Iancu, A. Leonidov and L. McLerran, Nucl. Phys. A 703 (2002) 489.

[4] M. A. Braun and C. Pajares, hep-ph/0405203.

[5] K. Golec-Biernat and M. Wuesthoff, Phys. Rev. D 59, 014017 (1999); D 60, 114023 (2000). 